\title{
Association between sagittal plane posture and quality of life in older people: a systematic review
}

\author{
Lívia Pimenta Renó Gasparotto1,2, Gláucia Regina Falsarella², Arlete Maria Valente Coimbra²
}

\begin{abstract}
Introduction: Changes in postural pattern during aging establish the body structural modifications that can compromise quality of life. The relationship between postural change and quality of life seems hardly mentioned among researches. Objective: The purpose of the article is to check on the subject's analyses, what are the possible associations should be highlighted in the preventive scope of the elderly with postural dysfunctions. Method: We used systematic review based on Pubmed and Lilacs by descriptors related to posture words and quality of life. The search was focused on articles published between 2005-2015. To analyze the quality and combination of data applied the STROBE checklist. Results: It identified 11 studies from which hyperkyphosis posture and lumbar adjustment were negatively associated with quality of life components. Conclusion: There was divergence in the direction of the association between lumbar concavity and quality of life. Although these observations contribute to the direction of preventive and corrective practices posture in aging, there is a need for more studies using mainly similar methodologies and homogeneous groups.
\end{abstract}

Key-words: posture, elderly, quality of life

\section{INTRODUCTION}

The aging process promotes several physical changes to the human body, among them, the changes in the postural pattern. Disorders such as loss of bone mass, muscle weakness and degenerative processes in the spine trigger the imbalance in the musculoskeletal structure, causing important deviations and compromising health. From the behavioral point of view, the postural addictions, the neglect of healthy habits, the sedentary life are verified as etiological factors. Processes such as these contribute to a disruption in the stability of the spine which causes unfavorable postures and possibly alter the quality of life indexes. ${ }^{(1-3)}$

The quality of life variables, present in several research instruments, have been shown to be an important tool for monitoring the health of the elderly, since they include information which includes the physical and psychological aspects of these individuals. However, the variability of its use for the analysis of different health-disease processes means that, despite its wide use, it is not widespread in certain subjects related to the health of the elderly. ${ }^{(5-7)}$

Knowing that the altered posture triggers changes in the musculoskeletal structure and that these correlate with impaired physical capacity, it seems reasonable to analyze the quality of life in these individuals, in order to quantify the impact of these misalignments on the conduct of life. In addition, a motor difficulty, caused by the restriction of movement originating from poor posture is also a precursory requirement of psychological aspects if considered its effects on autonomy and social relations..$^{(4,8)}$

The aim of this article is to evaluate, in the face of a systematic search, the articles which advocated between studies on the posture of the elderly, the use of the instrument of quality of life. In this way, all articles which applied any related instruments to the physical and psychological aspects present in the quality of life analysis will be selected and also those which focused on the angular characteristics of the sagittal plane postures.

\section{METHODS}

\section{Search strategy}

The most relevant articles of this research were identified from the databases: Pubmed and Lilacs. The search occurred for articles published in the last 10 years (2005-2015), written in the English and Portuguese languages, was limited. Thus, keywords were determined for the items related to the sagittal plane postures (kyphosis, kyphosis, lordosis, lordosis, posture, 
standing posture) and "quality of life". The words were used with the boolean operators AND and OR and flagged by Mesh Terms and Text Word.

The process of selection and analysis of articles was conducted in pairs. In cases of disagreement between the reviewers on the inclusion and exclusion criteria, the article was discussed to determine a consensus and then to decide whether or not to integrate the study into the research. The first selection was made based on the title of the articles. Then, in a second phase, the abstract of each article was analyzed in order to separate those which presented the required criteria for reading and revision. In case of unclear information, the authors of these articles were contacted and questioned about the data in question (figure 1).

\section{Inclusion and exclusion criteria}

The following inclusion criteria were determined: original articles aiming to identify the relationships between sagittal plane (hyperkyphosis and hyperlordosis) postures with quality of life; Studies published between January 2005 and December 2015; With elderly individuals over 65 years of age. Cross-sectional and longitudinal intervention studies were included in this study. In order to obtain greater statistical power in the results of association between variables, cross-sectional studies with more than 100 participants were preferred. In case of intervention studies, the minimum number of the sample should be 50 elderlies.

To quality of life analysis, the research was carried out using validated quality of life instruments, such as Short-Form Health Survey (SF-36) and Health Related Quality of Life (HRQoL), as well as researches which signaled the quality of life through data such as physical capacity, social and/or psychological aspects compatible with the information of the instruments mentioned above.

\section{Quality of selection}

To define the quality of data reported by transverse and observational articles, it was used the Strengthening the Reporting of Observational Studies in Epidemiology (STROBE) or STROBE initiative, according to Malta. ${ }^{\left({ }^{(9)}\right.}$ It consists of 22 required items to obtain greater accuracy in the description of these studies. These items are coded in zero (not contemplated item) or one (contemplated) indicating, therefore, the level of quality. The higher the score, the better the quality of the article. Each study was compared with the maximum score of this score. This strategy was determined to identify the highest quality studies (above $70 \%$ of the score), medium quality (between 50 to $69 \%$ ) and low quality (below $50 \%$ ). The instruments of evaluation for quality of life were described in figure 2 .

Consistency and direction of the association between Posture and Quality of Life
For the systematic review, the consistency of the association between posture and quality of life variables was identified. Consistency refers to the proportion of studies which presented posture association with the domains of quality of life. Consistent studies obtained a percentage above $60 \%$, moderate between 30 and $59 \%$ and inconsistent below $30 \%$. The percentage of studies indicating positive association was evaluated between the three types of study (transversal, longitudinal and intervention), while the direction identifies the association between these variables classified as positive, zero or negative.

\section{Identification of articles}

The following data were extracted from each article: country, sample type and size, ages of the participants, measurement instruments, variable adjustments.

\section{RESULTS}

The search resulted in 251 articles (232 articles extracted from Pubmed and 19 articles from Lilacs), according to figure 1. After reading the titles, all those which descriptions did not contain the determinants of the research (analysis of postural angles and their association with quality of life) were discarded.

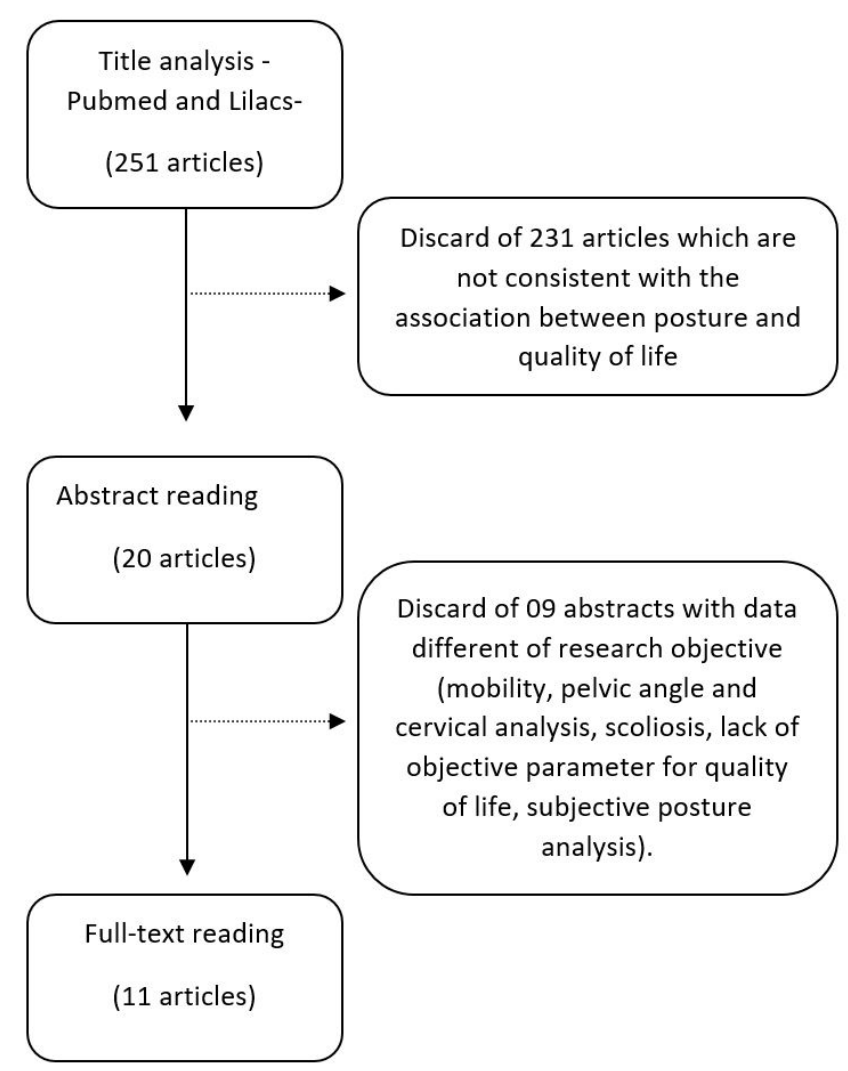

Figure 1. Systematic review flowchart 
QUALITY OF LIFE

Medical Outcomes Study 36- Item Short - Form Health Survey (SF-36): questionnaire comprising 36 items on eight components (domains): functional capacity, physical aspects, pain, general health, vitality, social functioning emotional aspects, mental health. ${ }^{(3)}$

Health Related Quality of Life (HRQOL): questionnaire consists of twelve fields of physical and psychological function functional status, vitality, social function, physical pain, emotions, general health, mental health. ${ }^{(10)}$

Mini Osteoporosis Quality of Life Questionnaire (mini OQLQ): consists of 10 items (abbreviated 30 items contained in the full questionnaire) among five domains: symptoms, physical function, activities of daily living, emotional function leisure. Used mainly to evaluate pharmacological and rehabilitation treatments in the elderly osteoporotic. ${ }^{(11,12)}$

Japanese Osteoporosis Quality of Life Questionnaire (JOQOL): evaluation instrument of quality of life in elderly Japanese with osteoporosis diagnostics. There are 38 items which score from 0 to 4 and may reach a maximum score of $152 . .^{(13)}$

Instrumental Activities of Daily Living (IADL): eight items assessing independence in daily living skills and quality of life aspects related to physical function. Scores range from 0 to 8 (dependent to independent) for women and 0 to 5 for men. Recommended instrument for use in non-institutionalized elderly group. ${ }^{(14)}$

POSTURAL EVALUATION

Radiography and Cobb method: Developed in 1948, it uses the radiography to calculate the curvature of the spine by means of the tangents of the vertebrae. In the sagittal plane postures, the tracings for the thoracic spine are observed between $\mathrm{T} 4$ and $\mathrm{T} 12$ or between the vertebrae of greater curvature in that plane. For the lumbar region, the traces starting from L1-L5, L1-S1, T12-S1 are observed. Although different tracing alternatives are noticed, this model (used in conjunction with the radiographic material) is considered a gold standard for postural evaluation, presenting good inter- and intra-rater reproducibility. ${ }^{(3,5,15,16,17,18)}$

Debrunner kyphometer: apparatus for measuring the column angle in which the bases are placed on spinous processes which it is desirable to analyze. Limited to work only on sagittal plane. ${ }^{(19)}$

Flexicurve: flexible ruler used to measure the angles of the spine on sagittal plane. The points $\mathrm{C} 7$ and T12 are located for the markings, the ruler is molded over the back, the column format is transcribed for the graph paper and the measurements are evaluated using an excel formula for angle identification. ${ }^{(10,20)}$

Inclinometer: easy-to-use instrument used to measure and tilt and elevation angles. There are pendulum-type models or embedded in compass gimbal. ${ }^{(14)}$

Spinal Mouse: unit formed by a curved frame and two wheels which measures the changes in inclination and their distances using an internal plumb. It communicates wirelessly with the base, which should be within two meters from the equipment. ${ }^{(7,15)}$

Figure 2. Used instruments in assessment of quality of life and body posture 
The main unrelated topics addressed surgical procedures, drug interventions, balance analysis, falls, and different age groups. Uncorrected securities totaled 231 and were discarded.

A total of 20 articles were defined for abstracts reading. After analysis, we also identified variables which do not address the objective specified in this review as a study of scoliosis, cervical angle, pelvic angles analysis, mobility, or study without objective posture analysis (questionnaire with questions and answers about having or not improper posture). Another did not point out objective determinants of quality of life and, finally, one was not a full article, just an editorial. In this stage 9 articles were discarded. At the end, 11 articles were defined for full reading, analysis and discussion.

\section{Quality of studies}

All articles scored (based on STROBE check-list) between 17 and 21 , which has a quality score between $77.2 \%$ and $95.4 \%$. The uncorrected STROBE checklist items were related to the lack of research funding description, measures taken to avoid bias, and characterization of the sample in results and methodology.

\section{General characteristics of the studies}

Among the eleven articles, one (9\%) is an intervention study and ten (91\%) are observational. The intervention study addresses the Yoga effects training on posture and quality of life. Among the observational articles, eight (80\%) are transversal and two (20\%) are longitudinal. Among transversals, one refers to the review article. Ten (91\%) contain a sample of elderly with participation of both sexes and only one (9\%) with exclusively male public. There were no studies with institutionalized elderly. Features such as osteoporosis, musculoskeletal disorders and vertebral fractures were also found in reading.

\section{Assessment of posture and quality of life}

The following measuring instruments for quality of life were identified: SF-36, HRQoL, mini-osteoporosis Quality of Life questionnaire (mini-OQLQ), Japan Osteoporosis quality of life (JOQOL), Instrumental Activities of Daily Living Scale (IADL).

To verify the posture, the used instruments (figure 2) were the Cobb method radiography, Debrunner kyphometer, Flexicurva and Spinal Mouse.

\section{Evidence of association between quality of life and posture}

Table 1 identifies the association of posture with domains (scores) of quality of life. Because the majority of analyzed articles do not specify each of components of quality of life, we opted for the analysis by physical and psychological score. These scores give a general score on the components relating to the physical and psychological aspects of the instruments. In addition, some articles dealt with quality of life using other parameters such as physical capacity, activities of daily living, satisfaction with life and socialization. These determinants were grouped in the model.

Studies have shown to be consistent. Among them, four (36.3\%) did not obtain significant results between posture data (hyperkyphosis and hyperlordosis) and quality of life. Another seven (63.7\%) presented some relation between the approached components. The direction of these relationships was generally negative with hyperkyphosis and quality of life, lumbar rectification and quality of life. This indicates that such deviations promote worsening of the living conditions of those people. However, regarding lumbar hyperlordosis, there were data indicating both positive and negative direction when analyzing its implication in quality of life components. It has also been observed that hyperkyphotic postures correlate with worse abilities in performing daily life activities (considered in one of the articles as a quality of life criteria).

Table 1. Direction and consistency of the association of quality of life scores with sagittal plane postures, in selected articles.

\begin{tabular}{|c|c|c|c|c|c|c|c|c|c|c|c|}
\hline \multirow{3}{*}{ QL domains } & \multicolumn{3}{|c|}{ Postural Change } & \multicolumn{6}{|c|}{ Association Direction } & \multicolumn{2}{|c|}{ Association Consistency } \\
\hline & \multirow{2}{*}{ TH } & \multirow{2}{*}{ LH } & \multirow{2}{*}{ LR } & \multicolumn{3}{|c|}{0} & \multicolumn{3}{|c|}{ - } & \multirow{2}{*}{$\%$ Total } & \multirow{2}{*}{ Classification } \\
\hline & & & & c & $\mathbf{L}$ & I & c & $\mathbf{L}$ & 1 & & \\
\hline \multirow{2}{*}{ Physical Score } & $1,5,7,9$ & 2,10 & 8 & $2,10,5$ & & & 7,8 & 1,9 & 11 & \multirow{2}{*}{$62.5 \%$} & \multirow{2}{*}{ consistent } \\
\hline & 10,11 & 11 & & & & & & & & & \\
\hline Physical aspects & & 2,3 & & & & & 2,3 & & & $100 \%$ & consistent \\
\hline Physical capacity & & 2,3 & & & & & 2,3 & & & $100 \%$ & consistent \\
\hline Activities of daily living & 6 & & & & & & 6 & & & $100 \%$ & consistent \\
\hline Psychological Score & $1,3,5,7,9$ & 11 & & & 1 & & $3,5,7$ & 9 & 11 & $66.6 \%$ & consistent \\
\hline Satisfaction with life & 4 & & & & & & 4 & & & $100 \%$ & consistent \\
\hline Socialization & 4 & & & & & & 4 & & & $100 \%$ & consistent \\
\hline
\end{tabular}

$\mathrm{QL}=$ Quality of life, $\mathrm{C}=$ cross-sectional studies, $\mathrm{L}=$ longitudinal studies, $\mathrm{l}=$ intervention studies, $\mathrm{TH}=$ thoracic hyperkinesis, $\mathrm{LH}=$ lumbar hyperlordosis, $\mathrm{LR}=$ lumbar rectification, $0=$ no association, - = negative association . 
Although these data point to a greater or lesser degree of association between the components of quality of life and postural changes, it is seen that some variables lose their significance because they are not relevant after regression analysis. Another limit observed is the different used instruments, which may compromise any consensus on several studies on the same subject, in the case, posture and quality of life.

\section{DISCUSSION}

Quality of life is widely used as a parameter of health in the elderly. The variability of the instruments allows its use in several dimensions of health, since it deals with functional, physical, psychological and social aspects. It is noted, among the instruments, some with applicability in the general population (SF-36, HRQOL) as well as those which are exclusive to the elderly public (Mini OQLQ, JOQOL). Although greater specificity is observed among some instruments, those applicable in the general population, such as SF-36, are very common in research with the elderly. $(2,3,10,21)$

The use of quality of life data in investigations in the area of aging consists in the fact that most diseases and comorbidities in this phase of life are subject to control and monitoring (the chronic diseases). Therefore, the daily quality of those who, at first, present one or more controllable conditions, becomes an important aspect of health. The instruments which assess quality of life are able to provide such information. ${ }^{(4,19)}$

Quality of life in the elderly seems to be influenced by the ability of the individual to perform routine activities. To have quality of life in the advancement of human aging means being independent for daily tasks and thus presenting satisfactory mental health. It is important that such a relationship is full of activity, autonomy and the least possible physical and emotional exhaustion. This fact corroborates the decision of some researchers to use the term quality of life through the results, found in the instruments, which evaluate the activities of daily living capacities. . $^{(3,4,10,18,19,21)}$

The process of human aging promotes physical wear to the spine. Disorders such as loss of bone mass in the spine region and head of the femur determine the intensity of osteoporosis by the level of bone fragility. Until recently, studies have stated that this was the main adverse characteristic of the spine which generated the postural deformity. It is justified by the researches with emphasis on the elderly osteoporotic and with vertebral fractures found in this review. Although such an assertion is considered, more recent studies have pointed out other etiologies for postural deviations such as muscle weakness. In these studies, elderly individuals with significant deviations and without diagnoses of osteoporosis were identified. ${ }^{(4)}$

In the review conducted, the Roux ${ }^{(10)}$ study worked with analysis of the quality of life in women diagnosed with osteoporosis in postmenopausal. In this study, which accompanied elderly women for three years, no relation to quality of life was identified in the first evaluation. Only after the third year there was posture correlation with the physical domains of quality of life. Imagama(3) related osteoporotic elderly of both genres with domains of quality of life. It was identified, in this respect, that those with compression fractures had the worst quality of life scores. The article points to a fact about vertebral fractures in women. Women with fractures and thoracic hyperkinesis are more vulnerable to death.

In the same line of action, Wang article $e^{(7)}$ classified the postural deviations between elderly Chinese with osteoporosis. It was identified among 450 volunteers a postural profile of kyphosis increase and reduction of lordosis. Thus, the relationship with quality of life was verified and it was observed that lumbar kyphosis is a negative predictor. In the sample presenting the postural type of hyperliphosis with hyperlordosis, it was noticed better scores on the quality of life.

The study Macintyre ${ }^{(14)}$ in elderly osteoporotic, it was identified the relationship between kyphosis and mobility test Time Up and Go. However, it was not found in those sample any relation in those individuals with pain.

It is seen among the references that the quality of life is more compromised in postmenopausal women and among the factors related, in addition to bone fragility, are the reduction in lumbar spine mobility and muscle weakness. This fact seems to be the same for men. ${ }^{(3)}$

Mobility is a recurrent term in articles regarding the posture and quality of life. It is not directly evaluated on any objective component of any questionnaire, but is prerequisite for items related to physical aspect. The lack of mobility, which generates restrictions of movement, prevents the elderly from successfully performing its activities. Thus, a column with important postural deviations necessarily alters muscle stability and this causes restricted angular movements between the spinal joints and limbs. ${ }^{(14)}$

The musculoskeletal system, specifically muscle weakness, is another factor that causes changes in postural balance, causing gravity to naturally sketch its strength while also propelling the spine anteriorly. This explains the fact that, when searching for references addressing quality of life and posture, not only articles with elderly osteoporotic but also without osteoporosis. These elderly people, for example, present significant postural deviation and obtain satisfactory results in the spine by only working on muscle strengthening. ${ }^{(2,4,19)}$

In this sense, the study of Greendale ${ }^{(19)}$ outlines the importance of yoga in the strengthening of muscles attached to the spine sustainability. After treatment, the kyphosis angle was reduced. However, the effect did not influence significant changes in quality of life after exercise intervention.

The study of Katzman ${ }^{(4)}$ on rehabilitation of elderly patients with kyphosis states that activities which specify the muscle 
rebalancing contribute to mitigate or reduce the deviation, since targeted.

Association of posture with quality of life was identified. However, the low number of researches, the variability of biotypes as well as the use of different instruments makes it difficult to standardize or specify such variables. Associations related to physical and emotional aspects were identified, to a greater or lesser extent, depending on gender and analysis type. In general, increased hyperkyphosis and reduction of lordosis seems to be negatively related to some determinants of quality of life. Mobility, although not included among components of quality of life, is understood as a factor to be considered because it has a direct impact on the physical aspects.

The article of Pelissè ${ }^{(11)}$ showed that the quality of life appeared worse for individuals who reported postural change than others with diagnoses of some chronic disease. However, it should be noted that the article did not include only elderly population, but individuals in general. Untreated chronic disease in the elderly may be determinant to a life of unfavorable conditions. Therefore, a standard for aging may not to be adopt by this obtained result in mixed populations.

Araújo ${ }^{(15)}$ identified the obesity and bone mass index as factors related to postural imbalance, but also did not obtain a homogeneous sample of elderly people. In another analysis, Araújo ${ }^{(16)}$ evaluated that the sagittal plane postures are not consistently associated with quality of life among men in the general population. When assessing the quality of life of women, the approach with postural imbalances occurred when the sacral inclination and pelvic parameters were added in the analysis, in addition to the sagittal plane postures.

The study Furuto and Yuki ${ }^{(8)}$ on elderly people found that men with increased kyphosis had lower total scores of the physical and mental components of quality of life. As for women with hyperkinesis, they had worse mental scores (psychological aspects).

The study of Macintyre ${ }^{(14)}$ found findings on quality of life and activity of daily living. The association between kyphosis and the Time Up and Go test was observed among elderly osteoporotic individuals, indicating impairment in the mobility of these individuals.

The study of Imagama ${ }^{(2)}$ indicates that the scores of the physical components of quality of life had a negative association with age and walking time of 10 seconds. In another analysis, the Imagama group ${ }^{(3)}$ identified relationship between lumbar lordosis, muscle strength and quality of life in the elderly and middle-aged men. However, when verifying multiple regression, only muscle strength obtained associative power.

The results on quality of life and postural parameters present some tendencies, but, they differ greatly from article to article in relation to the evidenced associations between postures and the studied population, in the case, the elderly individual. Considerations as biotype of the analyzed population (linked to ethnic factors), the used measurement instruments and the strategy of postural analysis should be highlighted as possible factors that trigger such divergences.

Regarding the postural changes, it is noted that older people increase the degree of kyphosis over the years and factors such as osteoporosis, vertebral fractures and muscle weakness are among the most cited items that, in certain cases, compromise the quality of life. The study of Roux ${ }^{(10)}$ with the use of strontium ranelate and the study of Greendale ${ }^{(19)}$ to yoga activity, demonstrated that these strategies have reduced the acceleration of kyphosis. Katzman ${ }^{(4)}$ indicates a prevalence of 20 to $40 \%$ thoracic kyphosis in the elderly population.

On the other hand, it does not seem to have the same pattern when analyzing lumbar lordosis. The study of Imagama ${ }^{(3)}$ and Wang $^{(7)}$ discuss the differences in the lumbar standard posture between different regions such as the USA and Japan. According to the region where these elderly people live, it may occur the reduce or progression of lumbar lordosis. In addition, the reduced lordotic curve seems to be related to worse resulting quality of life.

With respect to the measurement instrument, the presented literature does not indicate a standard. It is observed the use of inclinometer in the study of Macintyre ${ }^{(14)}$, Spinalmouse in the study of Wang ${ }^{(7)}$, the use of Debrunner kyphometer in Greendale studies ${ }^{(19)}$. The latter, because it is a question of pre and post-intervention evaluation, it is noted the use of different instruments at each moment. Furuto and Yuki( ${ }^{(8)}$ performed the postural analysis based on kyphosis index, which has the flexicurve rule as an evaluation object. The studies of Roux ${ }^{(10)}$, the two articles of Imagama ${ }^{(2,3)}$, the Schwab ${ }^{(18)}$, Smith $^{(5)}$ and Araujo studies ${ }^{(16,17)}$, they discussed their studies according to the $\mathrm{x}$-ray results, considered the gold standard for postural investigation. The instruments are used according to satisfactory approximation with the obtained results by $\mathrm{x}$-ray.

The paths between the vertebrae, which determine the inclination degree between each region of the spine are generally based on the idea provided by Cobb to establish measures strategies based on the radiograph. Note, however, the use of different measures for the same area of the spine in some studies, a fact that complicates the understanding of the real analyzed posture.

The hyperkyphosis analysis uses as defaults paths of T4 and T12, as studies of Roux ${ }^{(10)}$ and Katzman ${ }^{(4)}$. However, there are those which advocate the path in the two vertebrae that represent the largest local slope. The same occur for lumbar lordosis analysis, where the use of one study and another, alternate between L1-L5 or L1-S1, or even choose the overall alignment of the spine known as Roussouly method. ${ }^{(2,16)}$

\section{CONCLUSION}

It is observed, firstly, the lack of standardization among the methods for measuring the positions of sagittal plane. The variability of the methods may compromise the 
comparison among the results. Similarly, identification of life quality parameters linked to postural changes are hardly diffused in the literature. It is necessary further studies using these two components to increase the understanding level on the influence of the column positions in the quality of life of elderlies. At first there seems to be some tendencies to damage the physical aspects of quality of life for both genders, and regarding the psychological aspects, a greater interference in the male audience.

\section{AUTHORS' CONTRIBUTIONS}

LPRG participated in the project execution, systematic review and finalization of the article; GRF participated in the implementation of the project and review, AMVC participated in the design and structuring of the project as well as final review.

\section{CONFLICT OF INTEREST}

None

\section{REFERENCES}

1- Takahashi T, Ishida K, Hirose D. Trunk deformity is associated with a reduction in outdoor activities of daily living and life satisfaction in community-dwelling older people. Osteoporos Int 2005;16:273-279.

2- Imagama S, MatsuyamaY, Hasegawa Y, Sakai Y, Ito Z, Ishiguro N, et al. Back muscle strength and spinal mobility are predictors of quality of life in middle-aged and elderly males. Eur Spine J 2011;20:954-961.

3- Imagama S, MatsuyamaY, Hasegawa Y, Sakai Y, Ito Z, Ishiguro N, et al. Influence of sagittal balance and physical ability associated with exercise on quality of life in middle-aged and elderly people. Arch Osteoporos 2011;6:13-20.

4- Katzman WB, Wanek L, Shepherd JA, Sellmeyer DE. Age-related with hyperkyphosis: Its causes, consequences and management. J Orthop Sports Phys Ther 2011;40(6):352-360.

5- Smith J, Lafage V, Schwab F, Protocasats T, Keneberg E, Gupta M, et al. Prevalence and type of cervical deformity among 470 adults with thoracolumbar deformity. Spine 2014;39(17):1001-9.

6- Protopsaltis T, Schwab F, Bromsand K, Smith J, Klineburg E, Mundis $G$, et al. The T1 pelvic angle, a novel radiography measure of global sagittal deformity, accounts for both spinal inclination and pelvic tilt and correlates with health-related quality of life. J Bone Joint Surg Am. 2014;96(19):1631-40.

7- Wang H, Giambini H, Zhang W, Ye G, Zhao C, Na K, et al. A modified sagittal spine postural classification and its relatioship to deformities and spinal mobility in a chinese osteoporotic population. Plos One 2012;7(6):1-8.
8- Furuto J, Yuki M. Health-releated quality of life and self-efficacy for activities of daily living in elderly residents of mountainous areas with kyphosis: a one-year longitudinal study. Japanese Journal of Geriatrics 2014;(1):60-68.

9- Malta M, Cardoso LO, Bastos FI, Magnanini MMF, da Silva CMFP. Iniciativa STROBE: subsídios para a comunicação de estudos observacionais. Rev. Saúde Pública 2010;44(3):559-565.

10- Roux C, Fechtenbaum J, Kolta S, Said-Nahal R, Briot K, Benhamou CL. Prospective Assessment of thoracic kyphosis in postmenopausal women with osteoporosis. J of Bone and Mineral Res. 2010;25(2):362-368.

11- Pelisse F, Vila-Casadement A, Ferrer M, Domingo-Sabat M, Bago J, PirezGuieso $F$, et al. Impact on health related quality of life of adult spinal deformity (ASD) compared with other chronic conditions. Eur Spine J 2015;24(1):3-11.

12- Madureira MM, Ciconelli RM, Pereira RMR. Quality of life measurements in patients with osteoporosis and fractures. Clinics 2012;67(11):13151320.

13- Kumamoto K, Nakamura T, Suzuki T, Gorai I, Fujinawa O, Ohta H, et al. Validation of the Japanese Osteoporosis Quality of Lie Questionnaire. J Bone Miner Metab. 2010;28(1):1-7.

14- Macintyre Lorberos A, Adachi J. Inclinometer-based measures of standing posture in older adults with low bone mass are realible and associated with self-reported, but not performance-based, physical function. Osteoporos Int. 2014;25(2):721-8.

15- Suaide ALAP. Desenvolvimento e validação de uma ferramenta computacional para mensuração das curvaturas da coluna vertebral. Dissertação [Mestrado em Neurociências e comportamento], Instituto de Psicologia da Universidade Estadual de São Paulo, 2008.

16- Araujo F, Lucas R, Alegrete N, Azevedo A, Barros H. Individual and contextual characteristics as determinants of sagittal standing posture: a population-based study of adults. Spine J 2014;14(10):2373-83.

17- Araujo F, Lucas R, Alegrete N, Azevedo A, Barros H. Sagittal standing posture, back pain, and quality of life among adults from the general population: a sex-specific association. Spine 2014;39(13):E782-94.

18- Schwab F, Blondel B, Bess S, Hostin F, Shaffrey CL, Smith JS, et al. Radiographical spinopelvic parameters and disability in the setting of adult spinal deformity: a prospective multicenter analysis. Spine 2013;38(13):E803-12.

19- Greendale G, Huang M, Karlamangala A, Seeger L, Crawford S. Yoga decreases kyphosis in senior women and men with or without adult onset hyperkyphosis: results of a randomised controlled trial. J. Am. Geriatric Soc 2009;57(9):1569-1579;

20- Teixeira FA, Carvalho GA. Confiabilidade e validade das medidas da cifose torácica através do método flexicurva. Rev. Bras. Fisioter. 2007;11(3):199204;

21- Muszalik M, Kornatowski T, Wieczkowska HZ, Dijkstra KK. Functional assessment of geriatrics patients in regard to health-related quality of life (HRQOL). Clinical Interventions in Aging 2015;10:61-67; 